\title{
Dietary patterns and cardiovascular risk factors in young adults: the Young Hearts Project, Northern Ireland
}

\author{
H. J. McCourt ${ }^{1}$, C. R. Whittle ${ }^{1}$, I. S. Young ${ }^{1}$, S. J. Hunter ${ }^{2}$, L. J. Murray ${ }^{3}$, C. A. Boreham ${ }^{4}$, \\ A. M. Gallagher ${ }^{5}$, C. R. Cardwell ${ }^{3}$, J. V. Woodside ${ }^{1}$ and M. C. McKinley ${ }^{1}$ \\ ${ }^{1}$ Nutrition and Metabolism Group, Centre for Public Health, Queen's University Belfast, Belfast BT12 6BJ, UK, ${ }^{2}$ Regional \\ Centre for Endocrinology and Diabetes, Royal Victoria Hospital, Belfast BT12 6BA, UK, ${ }^{3}$ Cancer Epidemiology and Health \\ Services Group, Centre for Public Health, Queen's University Belfast, Belfast BT12 6BJ, UK, ${ }^{4}$ Institute for Sport and \\ Health, University College Dublin, Dublin, Ireland and ${ }^{5}$ Northern Ireland Centre for Food and Health (NICHE), University \\ of Ulster, Coleraine BT52 1SA, UK
}

Dietary pattern analysis, which reflects the complexity of dietary intake, has received considerable attention in nutritional epidemiology. It allows examination of the combined effects of nutrients and foods on markers of health ${ }^{(1)}$. Many studies have examined the relationship between dietary patterns derived by principle component analysis (PCA) and CVD risk factors in adult populations. Very few studies have examined these relationships at the adolescence or young adult stages.

The Young Hearts (YH) Project was designed to investigate CVD risk factors in a representative sample of 12-15-year olds in Northern Ireland ${ }^{(2)}$. Ethical approval was obtained from the Medical Research Ethical Committee of Queen's University Belfast and written informed consent was obtained from all participants ${ }^{(3)}$. The current findings are based on Young Hearts 3 (YH3), a follow-up examination conducted between October 1997 and October 1999 (mean (SD) age 22.6 (1.6), $n=489,51 \%$ males) ${ }^{(3)}$. 7-d diet histories were obtained by trained nutritionists and analysed for nutrient intake using Weighed Intake Software Program (WISP ${ }^{\odot}$ ) version 3.0 (Tinuviel Software, Warrington, UK). Fasting blood samples were analysed for: cholesterol fractions (total, HDL and LDL), TAG, plasma glucose and insulin, plasma total homocysteine, red cell folate and plasma vitamin $\mathrm{B}_{12}$. PCA was performed using SPSS version 17.0 to derive dietary patterns. Dietary patterns (split into quintiles) and risk factors for CVD were assessed by multivariate linear regression controlling for age, BMI, smoking status and energy intake.

Dietary patterns were identified separately for males and females (four patterns identified for each sex) but were named the same: 'Healthy', 'Traditional', 'Refined' and 'Social'. There was evidence to suggest that females who adhered most closely to a 'Traditional' dietary pattern had lower plasma total homocysteine $(P=0.005)$, higher red cell folate $(P<0.001)$ and higher serum vitamin $\mathrm{B}_{12}$ $(P=0.039)$ status. Similar findings were apparent for males who adhered most closely to a 'Healthy' dietary pattern: higher red cell folate $(P=0.013)$ and higher serum vitamin $\mathrm{B}_{12}(P=0.006)$ status. Males who adhered most closely to a 'Social' dietary pattern had a lower homeostatic model assessment score $(P=0.017)$.

Although previous studies in adults have reported associations between dietary patterns derived from PCA and several CVD risk factors, limited associations were observed in this sample. Young adulthood is recognised as a period of transition and dietary patterns may therefore be erratic and challenging to study at this life stage. Other methods of examining diet quality, such as ' $a$ priori' dietary scores, may be more useful at this stage in the life cycle and warrant further investigation.

The Young Hearts Project, Northern Ireland was supported by the Wellcome Trust and the British Heart Foundation.

1. Fung TT, Rimm EB, Spiegelman D et al. (2001) Am J Clin Nutr 73, 61-67.

2. Boreham C, Savage, JM, Primrose D et al. (1993) Arch Dis Child 68, 182-186.

3. Gallagher AM, Savage JM, Murray LJ et al. (2002) Public Health 116, 332-340. 